\title{
An integrated autonomous protection system against voltage instability based on Load Tap Changers
}

\author{
C. Vournas, C. Lambrou \\ School of Electrical and Computer Engineering \\ National Technical University of Athens, Greece
}

\begin{abstract}
In this paper we propose a local, completely autonomous protection system against voltage instability that can be readily integrated in the controller of each bulk power delivery LTC transformer. The protection system will identify an imminent voltage instability and will subsequently apply the necessary corrective measures to restore stable operation by properly adjusting the distribution voltage setpoint of the LTC. Application of this system on a 52-bus test system showed excellent performance for two unstable cases. For reasons of comparison, the indirect load curtailment proposed in a severe instability case is compared with direct load shedding schemes based on the local detection of instability, as well as on a global detection of instability assuming wide area measurements.
\end{abstract}

\section{Introduction}

In this paper the LIVES method (Local Identification of Voltage Emergency Situations) presented in [1-4] is used to design a completely autonomous protection system against voltage instability based on the LTC controller of bulk power delivery transformers. The method is based on monitoring the secondary (and eventually the primary) voltages on each LTC.

For signaling a voltage emergency alarm, the trend of the regulated, distribution-side voltage is monitored over a period of LTC operation (the time between successive operations). This should not be confused with the voltage response immediately after the tap adjustment. The latter changes direction when an impedance matching condition is met, as monitored for instance in [5-7]. This, however, occurs only after the onset of long-term voltage instability, as has been shown in [1]. In Section 2 of this paper various aspects of the LIVES method for issuing a voltage emergency alarm are summarized.

In [3] a simulated application of LIVES in the Hellenic Interconnected System showed that the method is in principle applicable to actual power systems and is quite

\author{
M. Glavic, T. Van Cutsem* \\ Dept. of Electrical Engineering and Computer Science \\ University of Liège, Liège, Belgium \\ * Fund for Scientific Research - FNRS
}

promising, but it demonstrated also some limitations of the LIVES approach. These include the effect of deadbands that may delay the emergency identification, as well as the effect of hard limits on the LTC available tap range. The latter disables the LTC operation, and thus the identification process on the corresponding buses.

Concerning the delay in identification, it should be noted that it coincides also with a delay in the actual manifestation of the instability. Thus it involves only a slight increase in the amount of corrective action, without hindering the protection performance. For the buses where LTCs have reached their control range limits, a special scheme was proposed in [4], which consists of a secondary voltage setpoint reduction, so as to restore LTC operation, and thus stability monitoring through the LIVES algorithm. It should be noted that the buses where LTCs reach their range limits are the ones with lowest transmission-side voltage, therefore those where it is most likely that an instability condition is identified first, and where a corrective measure would be most appropriate. This method is fine tuned in Section 3 of this paper to avoid unnecessary setpoint reduction, when the secondary voltage is increasing due to corrective actions in nearby buses.

In Section 4 of this paper we propose a novel method, in order to restore voltage stability following a LIVES alarm on an LTC. The countermeasure proposed is the reverse operation (in favor of transmission voltage) of the LTC for which the alarm is issued, until a condition of voltage stability is identified, at which point the setpoint of the distribution-side voltage is adjusted to its present value, thus providing a stable equilibrium point. The stability condition is monitored with a variant of the LIVES algorithm that we specify as "LIVES-restore" and is based on monitoring the primary (transmission-side) LTC voltage.

The autonomous protection system designed is simulated for the same test system (described in Section 5 of the paper) used in [2], based on the so-called Nordic32 system [8], and for the same unstable disturbances (Sections 6 and 7 respectively). It is seen that the method is effective and restores stability in both unstable cases. 
The indirect load curtailment accomplished by the distribution voltage setpoint reduction is compared to a protection system applying firm load shedding based on LIVES alarms. This amount of load shedding is also compared to the minimum amount necessary to restore stability. The latter is obtained using a centralized, global instability monitoring system (presented in [9-10] and described in Section 6B), which assumes accurate phasor measurements of the entire region subject to voltage instability.

\section{Local Identification of Voltage Emergency (LIVES-alarm)}

The LIVES method was originally presented in [1] and is based on monitoring the regulated secondary voltage of the Load Tap Changer (LTC) on each bulk power delivery transformer. As explained in [1-4], the tendency of this voltage to remain below the deadband even though the LTC is controlling, is a precursor of long-term voltage instability.

\section{A. Assumptions and sufficient stability condition}

Consider a power system whose long-term dynamics are those of the LTCs. The structure assumed for load bus $i$ is shown in Fig. 1. The variable ratio $r_{i}$ is on the primary (transmission) side and the LTC is controlling the secondary (distribution) voltage $V_{i}$. Load is considered voltage dependent. Short-term dynamics are assumed stable.

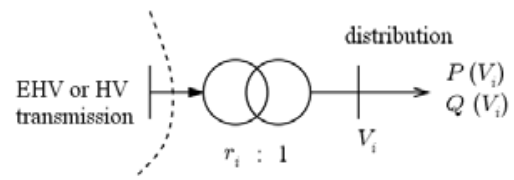

Figure 1: Transformer with LTC feeding voltage-sensitive load

Let $m$ be the number of LTC-controlled loads. The LTC mechanisms are discrete with a voltage deadband. Thus at every period of operation $T_{i}$ the $i$-th transformer ratio changes according to the difference equation:

$$
\begin{gathered}
r_{i}\left(k T_{i}\right)=r_{i}\left[(k-1) T_{i}\right]+\Delta r_{i}^{k} \\
\text { with } \Delta r_{i}^{k}=\left\{\begin{array}{ccc}
\Delta s_{i} & \text { if } & V_{i}>V_{i}^{\max } \\
0 & \text { if } & V_{i}^{\min } \leq V_{i} \leq V_{i}^{\max } \\
-\Delta s_{i} & \text { if } & V_{i}<V_{i}^{\min }
\end{array}\right.
\end{gathered}
$$

where $V_{i}^{\min }$ and $V_{i}^{\max }$ are the lower and upper deadband limits.

To facilitate the presentation, we assume that all LTCs have the same tap step and period of operation, i.e.

$$
\Delta s_{i}=\Delta s \quad T_{i}=T \quad \text { for } i=1, \ldots, m
$$

The general case of different tap step and time delay for each LTC can be handled as in [11]. The application study reported in this paper includes different time delay (period) for each LTC to show that this does not influence the validity of the results.

The stability of the discrete system (1) is guaranteed if all errors (distances from the deadband) are decreasing in absolute value at each period of operation [12]. Let us assume at first that after a severe disturbance all secondary voltages (at least in the area of interest) are below their respective deadbands. Then the LTC errors are defined as:

$$
e_{i}=V_{i}^{\min }-V_{i}>0, \quad i=1, \ldots, m
$$

Since by our assumption all errors are positive, they all reduce in absolute value when all secondary voltages increase after each period of operation. Thus, a sufficient stability condition under the assumptions outlined above is that:

$$
\Delta V_{i}^{k}=V_{i}\left(k T_{i}\right)-V_{i}\left[(k-1) T_{i}\right]>0, \quad i=1, \ldots, m
$$

In [2-3], the sufficient stability condition (5) is seen to be equivalent with stability conditions expressed in terms of the state Jacobian matrix of continuous or discrete systems. However, the LIVES method does not require any computation and is solely based on measurement and comparison of voltages after each LTC operation.

Violation of (5) at any LTC is an indication of approaching voltage instability. This condition can be monitored locally at each LTC, even though it is a system-wide indication as it indirectly involves all other tap changers operating with (approximately) the same time period. Thus, by monitoring the change of the secondary voltage (when it is below the deadband) on all LTCs, the onset of voltage instability can be detected in time to take corrective actions.

As long as the regulated voltages of all LTCs are increasing, the system is stable. LIVES alarm is based on the violation of condition (5), which is monitored locally on each LTC.

\section{B. Moving average and LIVES alarm algorithm}

The algorithm used in this paper for the identification of voltage instability and the issue of the alarm is based on moving average as described in [2]. The averaging helps 
to filter out fast transients and measurement noise. It is also used as an indirect record of the period just prior to a tap change.

The moving average of secondary voltage $V_{i}$ at a time $t_{j}$ is given by:

$$
\bar{V}_{i}\left(t_{j}\right)=\frac{1}{n_{i}} \sum_{k=0}^{n_{i}-1} V_{i}\left(t_{j}-k \Delta t\right)
$$

Where $\Delta t$ is the sampling period of the measurement and $n_{i}$ is the number of samples over which the moving average is calculated. The average is updated at each sampling instant $t_{j}=j \Delta t$.

The averaging period is taken equal to the corresponding LTC time delay $T_{i}$ to ensure that one and only one tap change of the LTC controlling the measured voltage is included in the average, when the LTC is active. In this way the averaging approximates correctly the general trend of $V_{i}$. Note that the averaging period is thus different for each measured voltage. The number of samples for each average is given by $n_{i}=T_{i} / \Delta t$.

With the assumptions made, the moving average immediately after a tap change occurring at $t_{k}=k T_{i}$ will vary by the following amount:

$$
\begin{aligned}
& \bar{V}_{i}\left(k T_{i}\right)-\bar{V}_{i}\left(k T_{i}-\Delta t\right)= \\
& =\frac{1}{n_{i}}\left[V_{i}\left(k T_{i}\right)-V_{i}\left(k T_{i}-T_{i}\right)\right]=\frac{1}{n_{i}} \Delta V_{i}^{k}
\end{aligned}
$$

with $\Delta V_{i}^{k}$ as defined in (5). Thus the sufficient stability condition is equivalent to an increasing moving average after a tap change:

$$
\bar{V}_{i}\left(k T_{i}\right)-\bar{V}_{i}\left(k T_{i}-\Delta t\right)>0
$$

Thus, an instant increase of moving average after a tap change is an indication of stability. On the other hand, if the moving average fails to increase after a tap change, this is an indication of an approaching instability. For security reasons, however, the alarm is not issued immediately but a time interval close to the period of LTC operation is allowed to pass, over which the moving average of the secondary voltage is being monitored.

Thus, while the controlled voltage is below the LTC deadband, the moving average at time $t_{k}$ of the $k$-th tap change is taken as a reference for monitoring the subse- quent evolution of the moving average:

$$
V_{r i}\left(t_{j}\right)=\bar{V}_{i}\left(t_{k}\right) \quad t_{j} \in\left[\begin{array}{ll}
t_{k} & t_{k}+T_{i}+\varepsilon
\end{array}\right]
$$

The term $\varepsilon$ can be tuned according to the application and the corrective measure to be used for facing the emergency. When a firm load shedding is performed, $\varepsilon$ should be positive for added security, i.e. in order to benefit from a longer observation period before shedding load. On the other hand, for the voltage stability restoration scheme described in Section $4, \varepsilon$ is taken as slightly negative to avoid one more tap change that will further degrade the system before the restoration process is activated.

The LIVES alarm detection process is as follows:

- If during the observation time the average voltage increases above the reference value (9), the counter is immediately reset and the process restarts after the next tap change;

- if the average voltage remains below the reference value (9) for a time $T_{i}+\varepsilon$, an alarm is signaled and the local voltage stability restoration starts.

It should be noted, that even though the violation of the sufficient condition (5) occurs before the onset of instability, due to the added time delay $\left(T_{i}+\varepsilon\right)$ the system will usually be already unstable for several seconds at the time the LIVES alarm is issued.

\section{Implementation aspects}

As described above, LIVES alarms are issued independently at each LTC controller using measurements already available in the controller itself. Actually all algorithms described in this paper can be easily programmed in existing LTC controllers.

When an alarm is issued, the corresponding LTC should clearly stop operating in its normal way following (2), as this will be ineffective due to the changed sign of (5) and it may initiate a voltage collapse. In the protection scheme propose in this paper the LTC controller starts an automatic voltage stability restoration process described in Section 4.

Even though the alarm and the voltage stability restoration are based entirely on local measurements, a minimum communication is necessary, in order to alert the Control Center that an emergency condition is encountered and that measures to restore voltage stability are being taken automatically. This is essential so that:

1. the voltage restoration action is not inadvertently stopped by local operators; 
2. the operators recognize the emergency and, if needed, start taking manual countermeasures according to emergency plans. These can include starting of local standby generation, curtailment of interruptible loads, manual switching of reactive devices, etc.;

3. normal operation is restored after the emergency conditions have passed.

One further point refers to the problem met in systems where some LTCs in the affected area are encountering their control range limits before a LIVES alarm is issued. This was already discussed in the Introduction of this paper and is a condition that was encountered in simulations of the Hellenic system [3]. The method to overcome this problem was originally proposed in [4] and consists of introducing a distribution voltage setpoint reduction each time an LTC is at its lower limit, while secondary voltage remains below the deadband. A new variant of this method is derived in this paper and is presented in Section 3 .

The added function of controllability restoration can also be incorporated in the LTC controller which will thus have 3 separate algorithms running:

1. LIVES-alarm during normal operation according to (2).

2. Range-limit monitoring and setpoint reduction to restore LTC operation.

3. Voltage stability restoration (LIVES-restore).

\section{LTC control range restoration}

Since LIVES method is based on measurements and comparison of secondary voltages after each LTC operation, it is essential for its functionality to restore LTC control range after they reach their hard limits.

To this end each LTC controller is programmed as follows:

- If after a tap change occurring at $t_{k}=k T_{i}$ the corresponding LTC reaches its lower tap limit, while the secondary voltage is below the deadband, the moving average at time $t_{k}$ is taken for a reference.

- If the moving average at $t=t_{k}+T_{i}$, where $T_{i}$ the corresponding LTC time delay, lies below the reference value, the distribution voltage setpoint is reduced by $5 \%$, otherwise (if the moving average is above the reference value) the current value of moving average is taken as a new reference and this step is repeated .

- The process stops when the secondary voltage returns inside its deadband.

The usage of the reference value in LTC control range restoration is necessary, in order to avoid setpoint reduction when the voltage starts to increase due to the coun- termeasures taken by the protection system applied, as will become evident in the application study of Section 7 .

The purpose of this module is to restore controllability of the secondary voltage and, most importantly, to allow the LIVES alarm algorithm to remain active so that a possible instability is identified and corrected. Of course the distribution voltage setpoint reduction is an indirect form of load curtailment, but it should be taken into account that (due to the LTC range exhaustion) the distribution voltage would decrease anyway and, what is worse, in an uncontrollable manner.

Clearly the restoration of controllability through setpoint reduction has to be communicated to the Control Center as a warning of possible upcoming voltage stability problems.

\section{Voltage stability restoration (LIVES- restore)}

After a LIVES alarm is issued on one load bus, any more tap changes in favor of distribution side voltage will produce the opposite result of further degrading all voltages including the controlled one. Thus voltage stability restoration is achieved by reversing the direction of LTC operation, i.e. by performing a series of tap changes in favor of the transmission-side voltage. This will initially increase the distribution-side voltage, due to the change of sign of (5), and will eventually increase also the transmission-side voltage, when stability conditions are restored.

The reverse LTC action should continue up to the point where voltage stability is restored. This condition, however, cannot be monitored through the successive changes of the secondary (distribution-side) voltage, because one of the assumptions made to obtain condition (5) is that all tap changes are made towards the same direction, while at the restoration phase LTC operation is reversed at each affected bus at a different time instant, while a number of other LTCs may still try to control the distribution voltage.

Thus, when an LTC is reversing its operation the corresponding error is assumed to decrease when the primary (transmission-side) voltage starts to increase. The corresponding stability condition (5) for this LTC thus becomes:

$$
\Delta V_{A i}^{k}=V_{A i}\left(k T_{i}\right)-V_{A i}\left[(k-1) T_{i}\right]>0, \quad i \in A
$$

where $V_{A i}{ }^{k}$ is the primary voltage and $A$ is the set of indices of LTCs that have reversed their operation after a LIVES alarm. 
As long as $\Delta V_{A i}{ }^{k}$ is negative, the system is still unstable, as at least the error of the reversing LTC is increasing. Thus the emergency situation persists and the reverse tap operation should continue. When, on the other hand, the primary voltage $V_{A i}{ }^{k}$ starts to increase under the combined effect of all taps, there is no longer an indication of violation of the sufficient stability conditions (5) and (10), and the emergency operation can be terminated. Note, however, that the increasing primary voltage is not a definite indication of stability, in case the normal LTC operation (2) is restored. This is a risk that can be taken, since after the normal LTC operation is resumed, LIVES alarm will be also available to monitor voltage stability. Thus, in the worst case, a cyclic operation may occur, but with the control center operators alerted, as discussed above, the emergency will be eventually alleviated.

Similarly to the emergency monitoring, a moving average of the primary voltage over a period of LTC operation is used and condition (10) is monitored through an increase of the moving average of the primary LTC voltage over a period $T_{i}+\varepsilon_{r}$. If the moving average of primary voltage remains above the corresponding reference for the period $T_{i}+\varepsilon_{r}$, a LIVES restore signal is issued and reverse tap operation stops.

Following this, the secondary voltage setpoint is lowered to its present value, in order to restore equilibrium at the current consumption level:

$$
V_{s i}=V_{i}(t)
$$

Note that the setpoint reduction corresponds to an indirect load shedding depending on the sensitivity of load to voltage shown in Fig. 1.

Let us assume at this point that the loads behind each LTC are exponential and that the active and reactive power consumed as a function of voltage are given by:

$$
\begin{aligned}
P_{i} & =P_{o i}\left(V_{i} / V_{o i}\right)^{\alpha} \\
Q_{i} & =Q_{o i}\left(V_{i} / V_{o i}\right)^{\beta}
\end{aligned}
$$

where $V_{o i}$ is the initial voltage, that is assumed to be the corresponding LTC setpoint and $P_{o i}, Q_{o i}$ are the active and reactive demands respectively.

When the secondary voltage setpoint is set to $V_{s i}$ the corresponding active and reactive power load curtailment is:

$$
\begin{aligned}
\Delta P_{i} & =P_{o i}\left[1-\left(V_{s i} / V_{o i}\right)^{\alpha}\right] \\
\Delta Q_{i} & =Q_{o i}\left[1-\left(V_{s i} / V_{o i}\right)^{\beta}\right]
\end{aligned}
$$

In the application section this load reduction will be compared to the theoretically minimum one for each specific unstable case.

\section{Test System description}

For reasons of comparison with [2], in this paper the same test system is used practically in the same Simulink-based environment. The test system is a variant of the so-called Nordic32 system detailed in [8]. The test system includes 52 transmission buses, 19 generators and one synchronous condenser, as shown in Fig. 2.

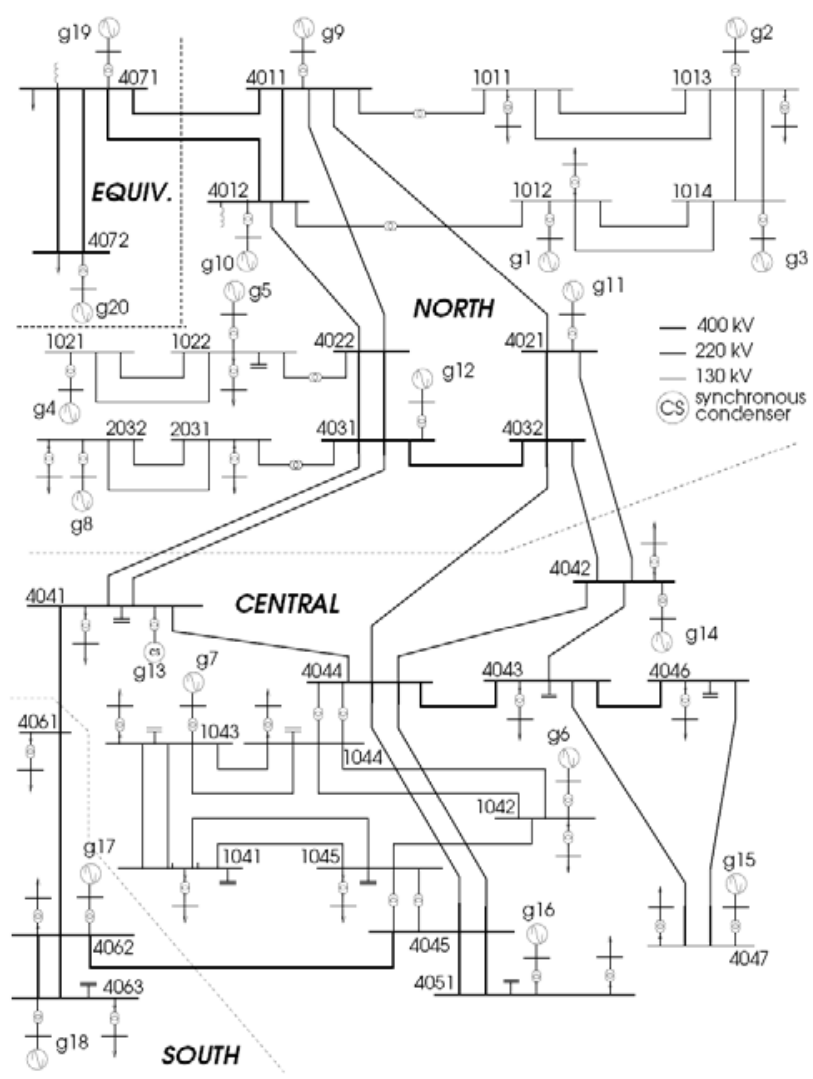

Figure 2: Nordic32 test system

As discussed in [2] two unstable and one stable case were examined. In all cases the disturbance simulated is the sudden loss of line 4032-4044 at $t=1 \mathrm{~s}$. In Case 1 the disturbance results in a severe voltage instability. In Case 2 loads in the base case are reduced, so that the system is marginally unstable after the same disturbance. Case 3 corresponds to a small further load reduction and is marginally stable. As seen in [2] no alarm is issued by the LIVES algorithm in this case, which is thus not reproduced in this paper, which deals with corrective measures following the identification of instability. 
System data are as reported in [2], with only minor changes in Overexcitation limiter (OEL) modeling. The model update allows the Automatic Voltage Regulator (AVR) to regain control, when the situation improves, but it results in slight changes in the exact time of OEL activation with respect to the results reported in [2], especially in Case 2.

The next two Sections deal with the performance of various protection schemes for the two unstable cases. The sampling period used to calculate the moving average is $\Delta t=50 \mathrm{~ms}$. In order to test robustness against measurement noise, Gaussian Noise with a mean value of 0 and a standard deviation of $10^{-3} \mathrm{pu}$ has been added to the sampled voltage. As explained in subsection $2 \mathrm{~B}$, the moving average is computed for each LTC over a time window equal to the delay between two successive tap changes.

\section{Case 1 (Severe Instability)}

\section{A. Simulation without protection scheme}

\begin{tabular}{|c|c|c|c|}
\hline t (s) & OEL & $\begin{array}{c}\text { LIVES } \\
\text { alarm }\end{array}$ & $\begin{array}{l}\text { Global } \\
\text { method }\end{array}$ \\
\hline 42.0 & g14 & & \\
\hline 43.7 & g15 & & \\
\hline 44.7 & & & Alarm \\
\hline 46.2 & g12 & & \\
\hline 50.5 & g7 & & \\
\hline 54.0 & & 4047 & \\
\hline 54.1 & & 1044 & \\
\hline 54.8 & g16 & & \\
\hline 55.1 & & 1041 & \\
\hline 57.0 & & 1045 & \\
\hline 57.1 & & 4042 & \\
\hline \multirow{2}{*}{60.0} & & 1043 & \\
\hline & & 4043 & \\
\hline 63.0 & & 4046 & \\
\hline 77.7 & g6 & & \\
\hline 85.6 & & 4051 & \\
\hline 92.0 & & 1042 & \\
\hline 92.1 & \multicolumn{3}{|c|}{ Voltage collapse } \\
\hline
\end{tabular}

In Case 1 described above, the contingency simulated initiates a voltage collapse in the central area of the system, as seen in Fig. 3. Voltages are slowly degrading and they eventually collapse at $\mathrm{t}=92.1 \mathrm{~s}$, due to the loss of synchronism of the field-current-limited generator g6. The sequence of events is shown in Table I. The first column gives simulation times, the second column the activation of OELs and the third the buses where LIVES alarms are issued.
In Table I the time for LIVES alarm is given for $\varepsilon=0$, i.e. after one period of LTC operation with the secondary voltage dropping.

\section{B. Direct load shedding based on LIVES alarm}

In this subsection we demonstrate the effectiveness of LIVES alarm to provide an instability warning early enough to apply corrective measures that will save the system from collapse. The suggested protection scheme consists of a direct load shedding at the bus, where the LIVES alarm is issued.

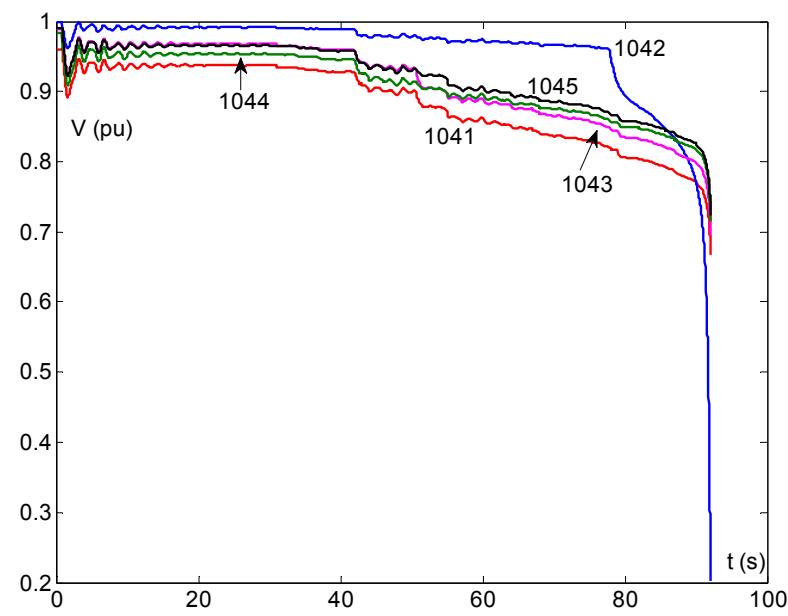

Figure 3: Evolution of voltages at HV buses (Case 1, no protection)

Due to the firm load-shedding action, in this case the delay for LIVES alarm is taken as $T_{i}+\varepsilon$, with $\varepsilon=1 \mathrm{~s}$ for all LTCs, while the load shedding is set to a constant percentage of the nominal load of each bus. Different percentage values are used for comparison.

Table II: Sequence of events for $10 \%$ direct load shedding and $\varepsilon=1 \mathrm{~s}$
\begin{tabular}{|c|c|c|c|}
\hline t (s) & OEL & $\begin{array}{c}\text { LIVES } \\
\text { Alarm }\end{array}$ & $\begin{array}{c}\text { Load } \\
\text { Shed- } \\
\text { ding } \\
(\text { MW) }\end{array}$ \\
\hline 55.0 & & 4047 & 10 \\
\hline 55.1 & & 1044 & 80 \\
\hline 56.1 & & 1041 & 60 \\
\hline 58.0 & & 1045 & 70 \\
\hline 58.1 & & 4042 & 40 \\
\hline \multicolumn{3}{|c|}{ Total shedding } \\
\hline
\end{tabular}

The sequence of events following the trip of line 40324044 at time $\mathrm{t}=1 \mathrm{~s}$ is shown in Table II for $10 \%$ load shedding after each LIVES alarm. The last two columns contain the bus at which the alarm occurs, along with the corresponding Load Shedding taken without any further delay. The events before the first alarm are not repro- 
duced in this and other Tables, as they are the same as in Table I.

As seen in Table II, the first bus where an emergency signal is issued is bus 4047 at $t=55 \mathrm{~s}$ and simultaneously $10 \mathrm{MW}$ of the load are shed. In the next seconds the protection scheme is activated in four more buses, leading to a total load shedding of $260 \mathrm{MW}$, which were enough to save the system, as can be seen in Fig. 4, which shows the voltages at $\mathrm{HV}$ buses in the affected central area with a $10 \%$ load shedding per LIVES alarm.

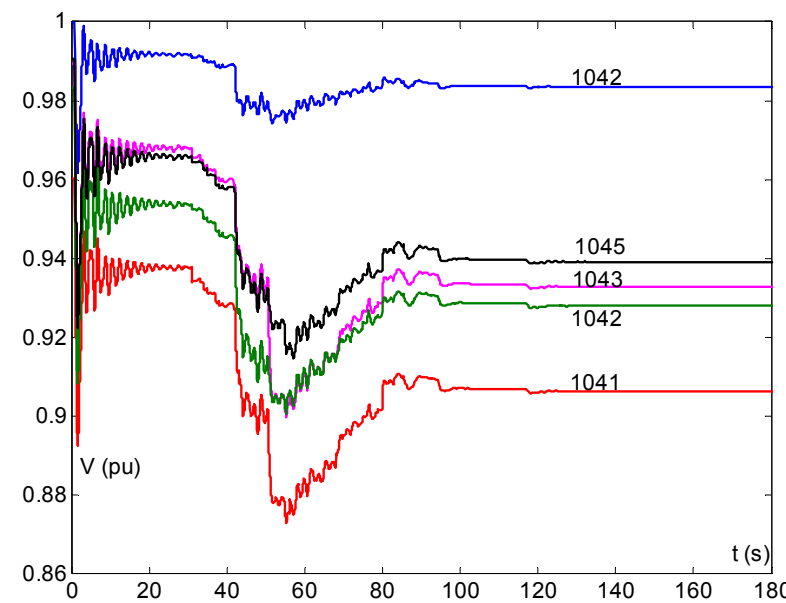

Figure 4: Transmission voltages for $10 \%$ direct load shedding (Case 1).

The procedure of the emergency detection and the subsequent load shedding are shown in Figs. 5 and 6 depicting the evolution of sampled secondary voltage, the moving average and the LTC ratio at buses 4047 and 1041 respectively.

As it can be seen from Fig. 5, the detection procedure at bus 4047 starts immediately after the first movement of tap at $\mathrm{t}=46 \mathrm{~s}$, by setting the reference value $V_{r}$ equal to the corresponding moving average. After this point, the average drops continuously below the reference value for the full LTC time delay of $8 \mathrm{~s}$ plus the additional $1 \mathrm{~s}$, and thus the emergency detection signal is issued at $t=55 \mathrm{~s}$ and a load shedding of $10 \mathrm{MW}$ is performed. Additional load shedding from neighbouring buses in the next seconds, results in a further increase of the secondary voltage, which comes inside the deadband at about $t=60 \mathrm{~s}$.

Another interesting case is that of bus 1041, shown in Fig. 6. At this bus the detection procedure starts at $t=31 \mathrm{~s}$, but due to the fact that the average increases above the reference value, the LIVES is reset until the next tap movement at $t=43 \mathrm{~s}$, where it starts dropping continuously below the new reference value $V_{r}$. The alarm and the corresponding load shedding are finally issued at $t=56 \mathrm{~s}$, since $T_{i}=12 \mathrm{~s}$.

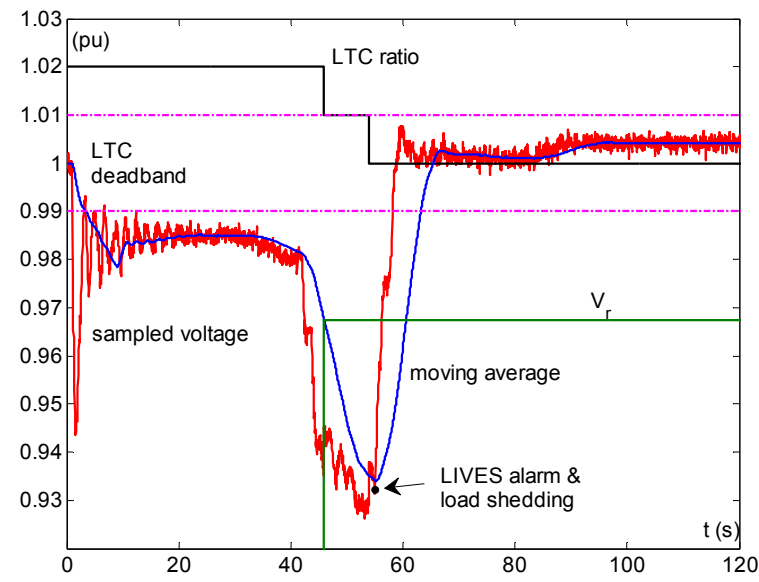

Figure 5: Voltage measurement, moving average, reference voltage and ratio at bus MV-4047 (Case 1)

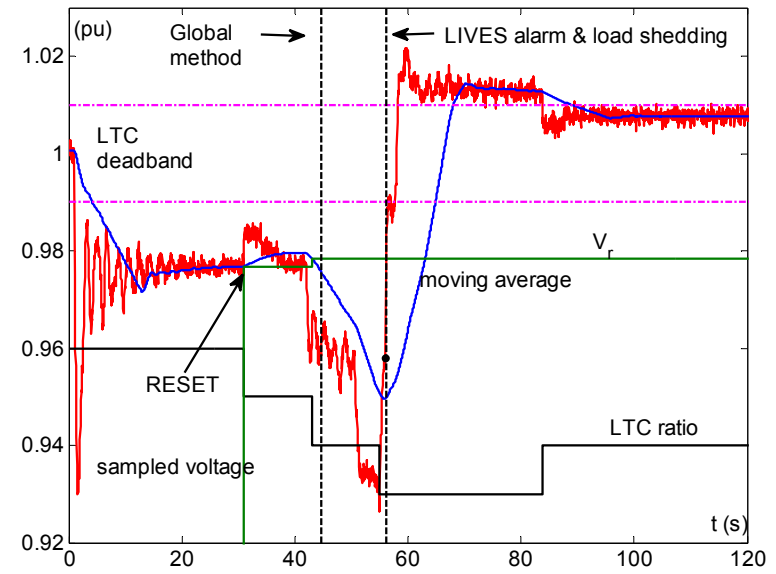

Figure 6: Voltage measurement, moving average, reference voltage and ratio at bus MV-1041 (Case 1)

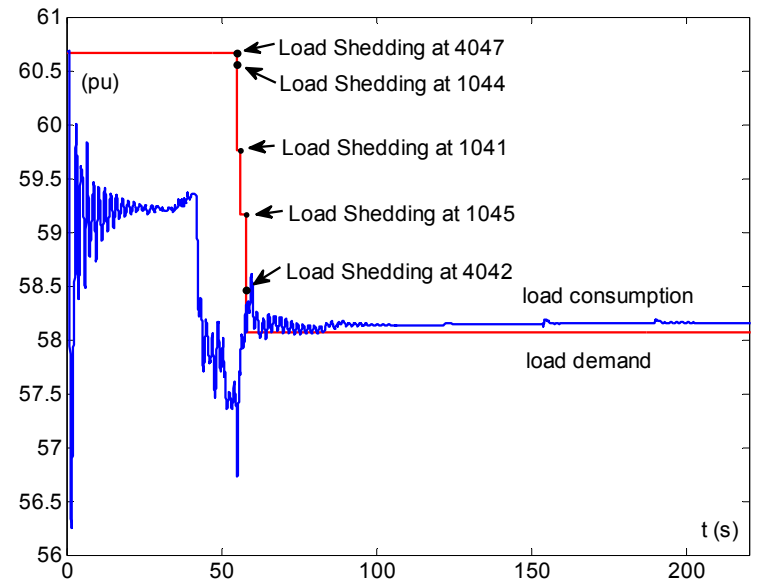

Figure 7: Total active load consumption and demand of the Central Area (Case 1)

In Fig. 7 the time response of the active load demand and consumption is shown. The system practically comes to a 
new stable steady state before $t=100 \mathrm{~s}$, with the load consumption satisfying the demand. The small deviation at the end is due to the effect of LTC deadband.

In general the total load curtailment is a function of the scheduled percentage of load shedding per LIVES alarm as shown in Fig. 8.

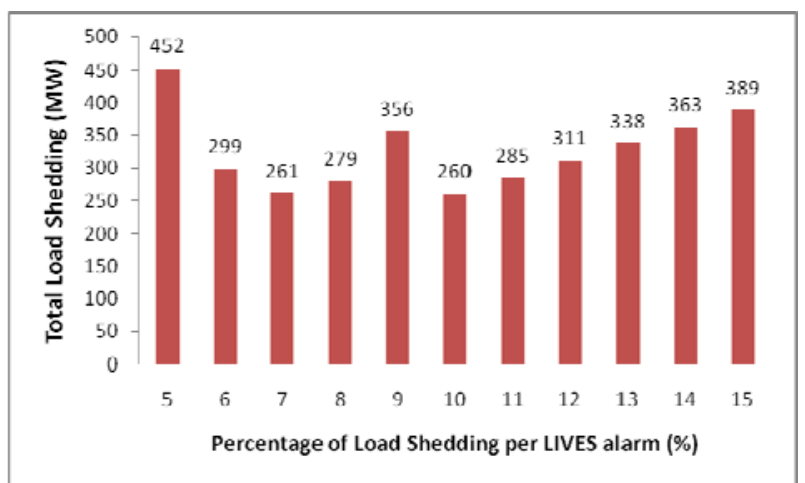

Figure 8: Total load shedding as a function of the load shedding per bus (Case 1)

The minimum load shedding percentage for the system to withstand the contingency is 5\%. For this smaller amount of load shedding, there are alarms in more buses and also repeated alarms at the same bus, as seen in Table III. When the percentage of load shedding increases up to $7 \%$, the total amount of shed load decreases, because fewer alarms are issued as seen in Table III. Note that generator g6 gets limited for the 5\% shedding, but its overexcitation is avoided for $7 \%$ load shedding, or more.

Table III: Sequence of events (Case 1) for 5\% and 7\% load shedding

\begin{tabular}{|c|r|r|r|r|r|r|}
\hline & \multicolumn{3}{|c|}{$\mathbf{5 \%}$} & \multicolumn{3}{c|}{$\mathbf{7 \%}$} \\
\hline t (s) & OEL & LIVES & MW & OEL & LIVES & MW \\
\hline 55.0 & & 4047 & 5 & & 4047 & 7 \\
\hline 55.1 & & 1044 & 40 & & 1044 & 56 \\
\hline 56.1 & & 1041 & 30 & & 1041 & 42 \\
\hline 58.0 & & 1045 & 35 & & 1045 & 49 \\
\hline 58.1 & & 4042 & 20 & & 4042 & 28 \\
\hline 61 & & 1043 & 12 & & 1043 & 16 \\
\hline 61 & & 4043 & 45 & & 4043 & 63 \\
\hline 85.7 & g6 & & & & & \\
\hline 88.0 & & 4046 & 35 & & & \\
\hline 92.0 & & 1041 & 30 & & & \\
\hline 122.6 & & 4051 & 40 & & & \\
\hline 152.0 & & 1041 & 30 & & & \\
\hline 179.6 & & 1042 & 15 & & & \\
\hline 180.3 & & 1044 & 40 & & & \\
\hline 180.5 & & 4046 & 35 & & & \\
\hline 181.9 & & 4047 & 5 & & & \\
\hline 182.7 & & 1045 & 35 & & & \\
\hline \multicolumn{2}{|l|}{ Total shedding } & $\mathbf{4 5 2}$ & & & \\
\hline
\end{tabular}

When the load shedding percentage is set to $8 \%$ and above, the total load curtailment starts to increase. However, for a load shedding percentage of $10 \%$, shedding at buses 1043 and 4043 is avoided as can be seen by comparing Tables II and III, and thus the total load-shedding amount is reduced. For shedding percentages above $10 \%$, the buses at which LIVES alarms are issued remain unchanged (with a single alarm per bus), and thus the total load shedding increases monotonically with the loadshedding percentage.

\section{B. Minimum load shedding}

The load curtailment achieved with the voltage stability protection method proposed in the previous subsection $6 \mathrm{~A}$ is compared here to the minimum load shedding necessary to restore stability for the unstable scenario of Case 1 .

The minimum necessary load shedding is computed based on the early detection of impending voltage instability method described in [9-10]. This method assumes that the region prone to voltage instability is equipped with PMUs ensuring full observability of bus voltages and performs an efficient sensitivity computation in order to identify when a combination of load powers has passed through a maximum.

Since this method is based on global information it is able to identify the instability at the time the maximum loading conditions occur. In the simulation of Case 1 the voltage instability identification is made at time $t=44.7 \mathrm{~s}$. As seen in Table I, the detection using the global method is earlier than the first LIVES alarm. This is due to the security time delay of $T_{i}$ introduced for detecting the instability by the LIVES method. Thus, even though the condition monitored by LIVES is violated before the onset of instability (at $t=43 \mathrm{~s}$ as seen in Fig. 6) the detection is made a few seconds later.

The minimum load shedding is performed immediately at the time of instability detection based on the sensitivities of the most affected load voltage to load changes at each bus [13]. These normalized sensitivities (weighted active and reactive power load) are shown in Table IV. We assume the maximum load shedding on each bus to be equal to $20 \%$ of bus load. A larger percentage of load rejection may be infeasible and also, since the sensitivities are based on linearization, larger percentages of load shedding are likely to influence the ranking of Table IV.

The calculation of the minimum necessary amount of load shedding to restore stability is based on repeated calculations for an increasing amount of load shed until a stable response is achieved. Load is shed first from the bus with 
the highest sensitivity, up to the maximum permissible percentage and the simulation is repeated until a stable response is achieved.

Table IV: Normalized sensitivities of each load

\begin{tabular}{|c|c|r|}
\hline Bus & Sensitivity & $\begin{array}{c}\text { Load Shed } \\
\text { (MW) }\end{array}$ \\
\hline 1041 & 1.000 & 120 \\
\hline 1043 & 0.934 & 48 \\
\hline 1044 & 0.732 & 30 \\
\hline 4042 & 0.714 & \\
\hline 1045 & 0.690 & \\
\hline 4043 & 0.587 & \\
\hline 4046 & 0.547 & $\mathbf{1 9 8}$ \\
\hline 4047 & 0.500 & \\
\hline 4051 & 0.452 & \\
\hline 1042 & 0.300 & \\
\hline \multicolumn{2}{|c|}{ Total shedding } & \\
\hline
\end{tabular}

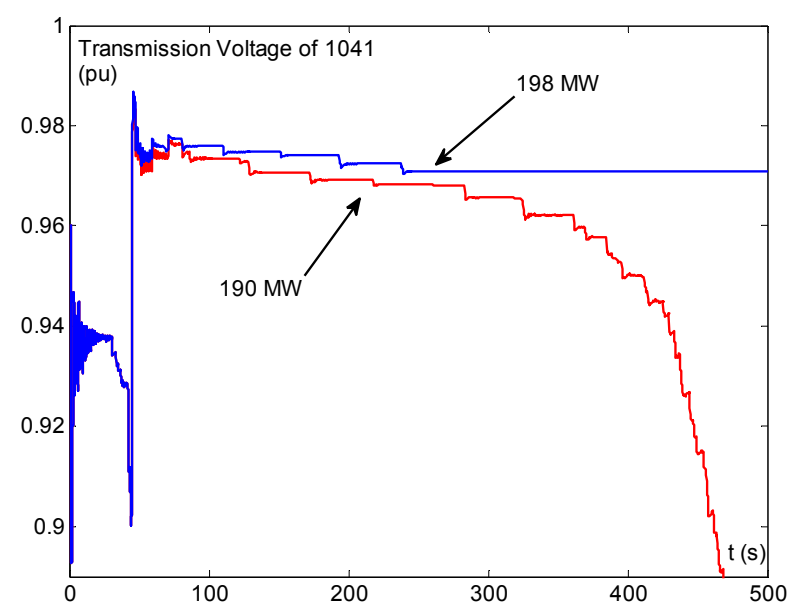

Figure 9: Determination of minimum load shedding based on sensitivities (Case 1)

The marginally stable and unstable simulation cases are shown in Fig. 9. As seen the minimum load shedding in Case 1 is $198 \mathrm{MW}$, which is less than that achieved in the previous subsection 6A. This is to be expected since the load shedding starts earlier and is optimized as load is shed first from the most efficient bus.

Another important remark is that the order of buses where LIVES alarms are issued, even though it depends on more or less random factors, such as the assumed time delay of each LTC, is not so different from the most effective one based on sensitivity calculations. For instance 4 out of the 5 most effective buses for load shedding of Table IV are included in the 5 first buses where LIVES alarms are issued, as seen in Tables I-III. This selectivity property of the LIVES method is inherent, since the buses most affected by the instability (and thus the first to identify emergency conditions) are also in general the ones most effective for countermeasures.

\section{Voltage Stability Restoration (LIVES-restore)}

In this subsection, instead of firm load shedding we apply after each LIVES alarm the Voltage Stability Restoration process described in Section 4. For this application the time window for computing the LIVES alarm $\left(T_{i}+\varepsilon\right)$ is taken slightly smaller than $T_{i}$ (the time delay of the $i$-th LTC), i.e. $\varepsilon$ is set to a small negative number $(-0.1 \mathrm{~s})$, in order to inhibit an unnecessary movement of the LTC in the wrong direction before starting the reverse operation immediately after the alarm. The voltage stability restoration by reverse tapping continues until the moving average of primary side voltage is increasing over a time pe$\operatorname{riod} T_{i}+\varepsilon_{r}$, with $T_{i}$ the same as previously and $\varepsilon_{r}=1 \mathrm{~s}$.

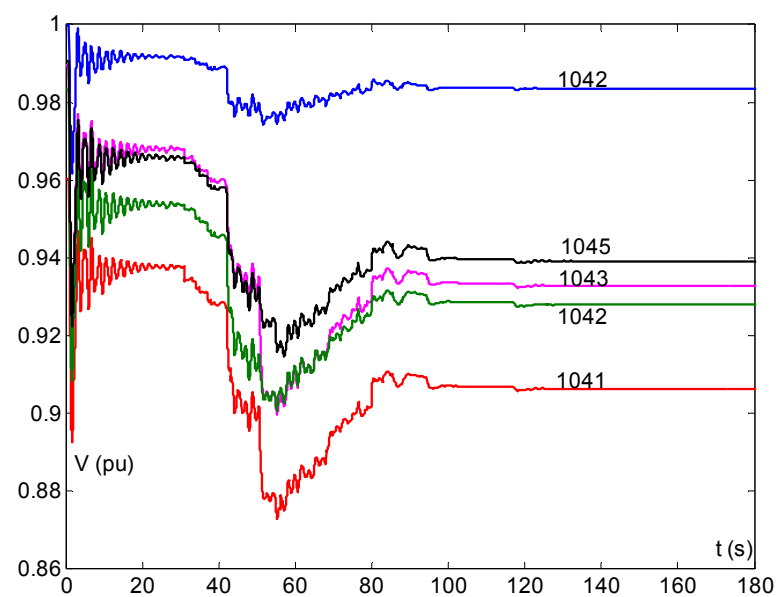

Figure 10: Voltage in transmission buses with LIVES-restore (Case 1)

The responses of HV bus voltages with voltage stability restoration are plotted in Fig. 10, while the sequence of events is shown in Table V. Small differences exist in the sequence of LIVES alarm with respect to previous Tables, due to the difference of the additional time $\varepsilon$. LIVES alarms are issued in more buses than in the case of firm load shedding.

As seen, LIVES-restore signals appear between $t=71.6 \mathrm{~s}$ and $t=89.1 \mathrm{~s}$. After each LIVES-restore action the secondary voltage setpoint is reduced to its current measured value. As explained in Section 4, this corresponds to an indirect load shedding, which is shown in the fifth column of Table V. The total amount of indirect load shedding in this case is $281.5 \mathrm{MW}$.

The performance of the proposed integrated protection scheme (LIVES-alarm and LIVES-restore) can be assessed by examining the MV and HV voltages at bus 1041, which are shown in Figs. 11 and 12 respectively. 
When LIVES alarm is issued, at $t=55 \mathrm{~s}$, immediately the reverse tap operation starts and the detection for LIVESrestore conditions is enabled.

\begin{tabular}{|c|c|c|c|c|}
\hline $\mathbf{t}(\mathbf{s})$ & OEL & $\begin{array}{c}\text { LIVES } \\
\text { alarm }\end{array}$ & $\begin{array}{l}\text { LIVES } \\
\text { restore }\end{array}$ & $\begin{array}{l}\text { Indirectly } \\
\text { shed load } \\
\text { (MW) }\end{array}$ \\
\hline 53.9 & & 4047 & & \\
\hline 54.0 & & 1044 & & \\
\hline 54.8 & g16 & & & \\
\hline 55 & & 1041 & & \\
\hline 56.9 & & 1045 & & \\
\hline 57.0 & & 4042 & & \\
\hline & & 1043 & & \\
\hline 59.9 & & 4043 & & \\
\hline 62.9 & & 4046 & & \\
\hline 71.6 & & & 4047 & 7.7 \\
\hline 76.0 & & & 1044 & 53.1 \\
\hline 78.9 & & & 4042 & 30.1 \\
\hline 79.8 & & & 1043 & 14.3 \\
\hline 81.0 & & & 1045 & 40.5 \\
\hline 81.2 & & & 1041 & 38.1 \\
\hline 84.0 & & & 4043 & 56.7 \\
\hline 89.1 & & & 4046 & 41.0 \\
\hline \multicolumn{4}{|c|}{ Total load curtailment } & 281.5 \\
\hline
\end{tabular}

After the first reverse movement of LTC tap ratio, the protection controller is monitoring the transmission-side voltage of the bus, as shown in Fig. 12. Since this voltage tends to increase for a period equal to LTC delay plus $\varepsilon_{r}$, it is deduced that voltage stability conditions are met. At this point LIVES-restore issues a signal to reduce the setpoint of the secondary voltage at its measured value (Fig. 11).

With this integrated protection method the system was able to withstand successfully the severe contingency, as seen in Fig. 13, where the load demand and load consumption are shown.

\section{Discussion}

As seen in this section, the emergency identification through LIVES-alarm with the delay implemented is early enough to apply voltage stability protection. The firm load shedding based on LIVES alarm can be comparable with the theoretically minimum amount obtained using a method of global identification by assuming observability of the whole region. This minimum amount was approximated by less than $60 \mathrm{MW}$.

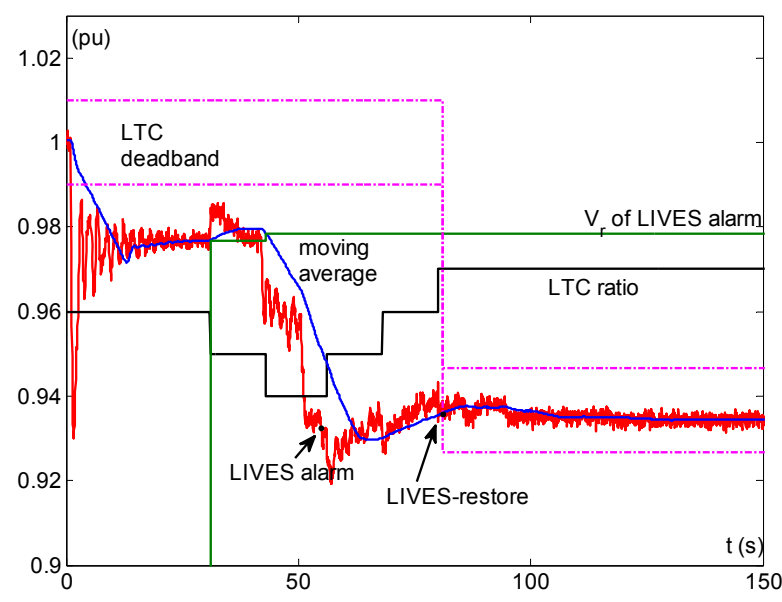

Figure 11: Voltage measurement, moving average, reference voltage and ratio at MV side of bus 1041 (Case 1)

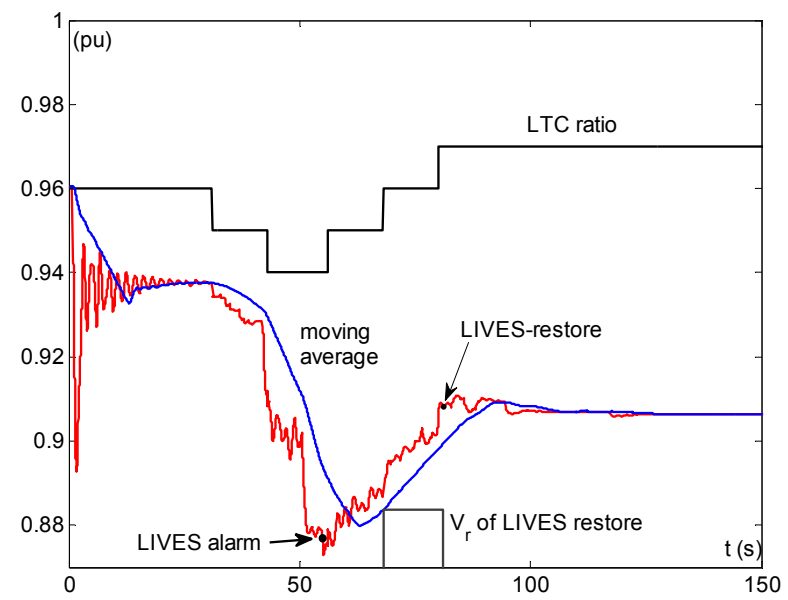

Figure 12: Voltage measurement, moving average, reference voltage and ratio at $\mathrm{HV}$ side of bus 1041 (Case 1)

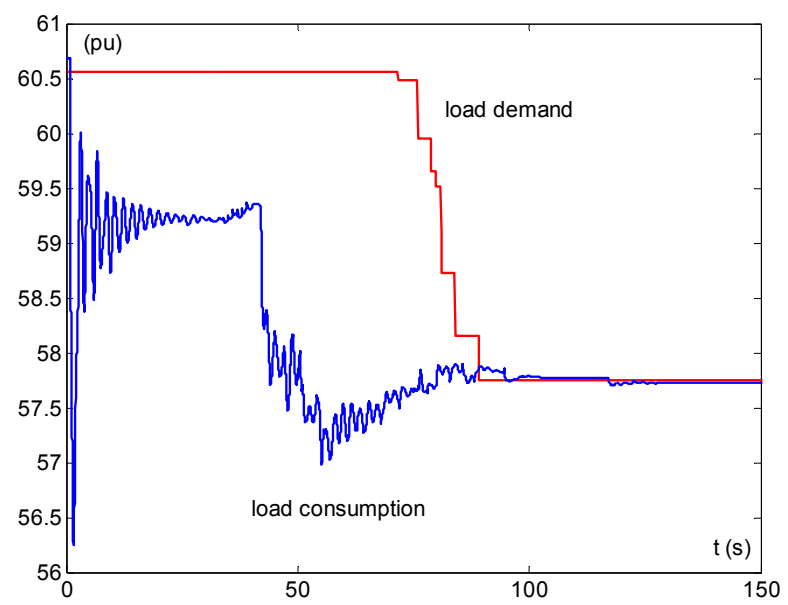

Figure 13: Total active load consumption and demand of the Central Area (Case 1, integrated protection scheme) 
Compared to firm load shedding, the integrated voltage stability restoration method based on reverse tapping and distribution voltage setpoint reduction is preferable for the following reasons:

1. it is a softer measure from the consumers' point of view;

2. it is straight forward to implement solely on the LTC controller without the need for wiring and telecommunications;

3. there is no need for a predefined percentage of load shedding.

On the other hand, the disadvantage of LIVES-restore is that setpoint reduction is not safe when a downstream load restoration mechanism is active, as would be the case with distribution voltage regulators and thermostatic loads. However, it is reasonable to expect that in this case the method would still keep the system up long enough for operators to take other permanent corrective actions, as discussed in subsection 2C. Another cost of this less intrusive load curtailment is that it requires a slightly larger amount of load loss, in this case another $20 \mathrm{MW}$ more than in the minimum load shedding based on LIVES alarms, or $80 \mathrm{MW}$ based on the theoretical minimum load shedding.

\section{Case 2 (Marginal Instability)}

\section{A. Simulation without protection scheme}

As in Case 1, we start by presenting first the results of the simulation without protection. In this case the base-case load is lower, so the instability takes longer to manifest itself, as shown in the succession of events of Table VI.

Table VI: Sequence of events in Case 2, with no protection
\begin{tabular}{|c|c|c|}
\hline t (s) & OEL & $\begin{array}{c}\text { LIVES } \\
\text { alarm }\end{array}$ \\
\hline 85.6 & $\mathrm{~g} 7$ & \\
\hline 92.0 & $\mathrm{~g} 14$ & \\
\hline 402.4 & $\mathrm{~g} 15$ & \\
\hline 463.4 & $\mathrm{~g} 16$ & \\
\hline 507.3 & $\mathrm{~g} 12$ & \\
\hline 510.1 & & 1041 \\
\hline 527.2 & & 4046 \\
\hline 532.6 & & 4051 \\
\hline 534.4 & & 4047 \\
\hline 540.6 & & 1043 \\
\hline 541.1 & & 1044 \\
\hline 549.0 & g6 & \\
\hline 550.0 & & 4042 \\
\hline 551.2 & & 4043 \\
\hline 564.2 & & 1042 \\
\hline 565.6 & Voltage collapse \\
\hline
\end{tabular}

For eight minutes after the disturbance the LTCs are barely able to regulate the secondary voltages, but in the absence of countermeasure the system collapses at $t=565.6 \mathrm{~s}$, as seen in Fig. 14 showing the active load consumption and demand in the affected Central area.

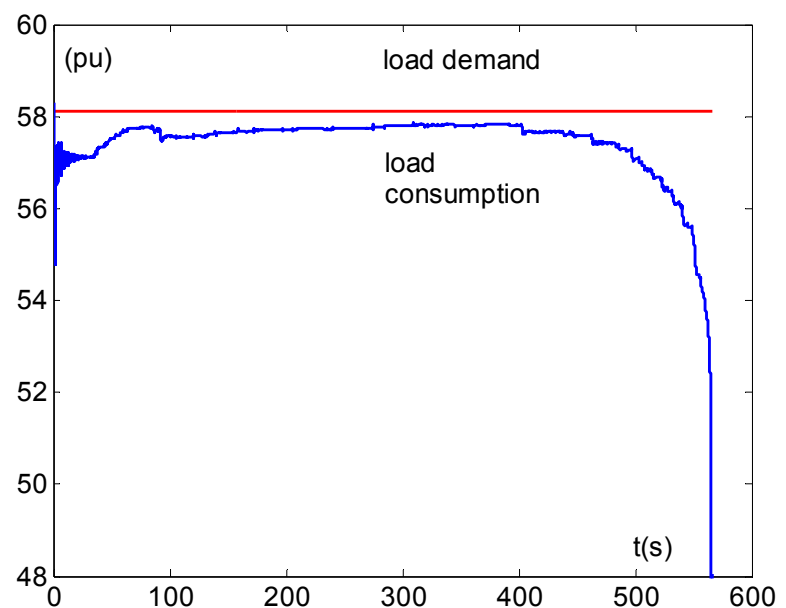

Figure 14: Total active load consumption and demand of the Central Area, Case 2 with no protection scheme.

As the comparison with other load shedding schemes has already been made in Case 1 , in this case we will only consider the integrated protection scheme of stability restoration by reverse taps and distribution setpoint reduction.

\section{B. Voltage Stability Restoration (LIVES-restore)}

The voltage instability detection and the subsequent stability restoration with LIVES-restore method is shown in Fig. 15, where the secondary (MV) voltage of bus 1041 is plotted. The sequence of events for this protection scheme is shown in Table VII. In the second column of this Table, OEL means that the overexcitation limiter of the corresponding generator is activated, while AVR means that the OEL is reset and AVR operation is restored.

It is clear from Fig. 15 that the LIVES-alarm process resets every time the LTC brings the secondary voltage inside its deadband, until $t=510 \mathrm{~s}$, at which time a LIVES alarm is issued. Then the reverse action of LTC is enabled, and LIVES-restore is monitoring the primary side of bus 1041, shown in Fig. 16, until at $t=572.1 \mathrm{~s}$ a LIVES-restore signal is issued. The corresponding setpoint reduction causes an indirect load shedding at this bus of about $48.4 \mathrm{MW}$. The total indirect load shedding, achieved by the protection scheme in Case 2 is about 165.6 MW as seen in Table VII. 
Table VII: Sequence of events in Case 2 with LIVES-restore

\begin{tabular}{|c|c|c|c|r|}
\hline t (s) & $\begin{array}{c}\text { Gen } \\
\text { OEL \& } \\
\text { AVR }\end{array}$ & $\begin{array}{c}\text { LIVES } \\
\text { alarm }\end{array}$ & $\begin{array}{c}\text { LIVES } \\
\text { restore }\end{array}$ & $\begin{array}{c}\text { Indirectly } \\
\text { shed load } \\
\text { (MW) }\end{array}$ \\
\hline 85.6 & g7 OEL & & & \\
\hline 92.0 & g14 OEL & & & \\
\hline 402.4 & g15 OEL & & & \\
\hline 463.4 & g16 OEL & & & \\
\hline 507.3 & g12 OEL & & & \\
\hline 510.0 & & 1041 & & \\
\hline 542.3 & & 4047 & & \\
\hline 545.0 & & 1045 & & \\
\hline 550.0 & & 4051 & & \\
\hline 551.1 & & 4043 & & \\
\hline 551.1 & & 4046 & & \\
\hline 556.9 & g6 OEL & & & \\
\hline 560.1 & & & 4047 & \\
\hline 569.1 & & & 1045 & \\
\hline 570.3 & & & 4051 & \\
\hline 572.1 & & & 1041 & \\
\hline 575.1 & & & 4043 & \\
\hline 576.2 & g6 AVR & & & \\
\hline 577.3 & & & 4046 & \\
\hline 727.1 & g16 AVR & & & \\
\hline 746.1 & g15 AVR & & & \\
\hline 759.3 & g12 AVR & & & \\
\hline 813.8 & g7 AVR & & & \\
\hline 814.1 & g14 AVR & & & \\
\hline & \multicolumn{2}{|c|}{ Total load curtailment } & \\
\hline
\end{tabular}

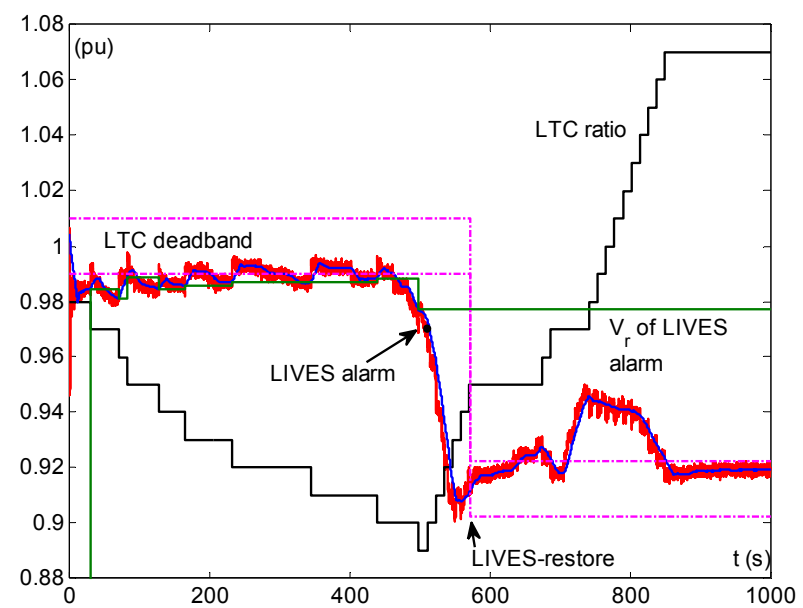

Figure 15: Voltage measurement, moving average, reference voltage and ratio at MV side of bus 1041 , Case 2

\section{Operation of LTC control range restoration module}

In this marginally unstable case, due to the long time before the occurrence of instability, two LTCs reach their lower tap limit (88\%) as shown in Table VIII, which lists the lowest tap ratio for each LTC of the central area.

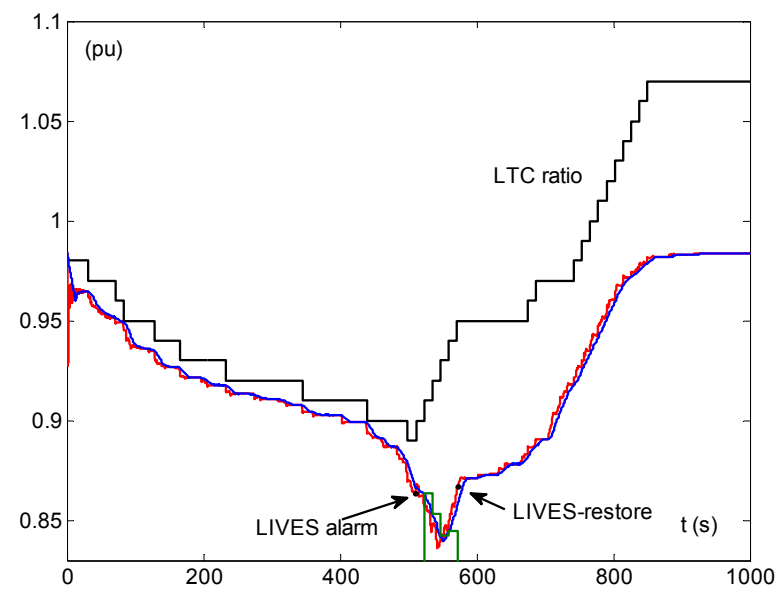

Figure 16: Voltage measurement, moving average, reference voltage and ratio at $\mathrm{HV}$ side of bus 1041 , Case 2

Table VIII: LTC lowest ratio, Case 2
\begin{tabular}{|c|c|}
\hline Bus & $\begin{array}{c}\text { LTC lowest } \\
\text { ratio }\end{array}$ \\
\hline 1041 & 0.89 \\
\hline 1042 & 0.98 \\
\hline 1043 & 0.88 \\
\hline 1044 & 0.88 \\
\hline 1045 & 0.91 \\
\hline 4041 & 0.96 \\
\hline 4042 & 0.92 \\
\hline 4043 & 0.93 \\
\hline 4046 & 0.91 \\
\hline 4047 & 0.97 \\
\hline 4051 & 0.91 \\
\hline
\end{tabular}

The operation of the LTC range restoration function described in Section 3 monitored the range limit condition, but the setpoint reduction to restore the control range was not activated, because the secondary voltage (even though below deadband) was found to increase due to the voltage stability restoration process at nearby buses. This is clearly seen in Fig. 17, showing the LTC tap ratio and secondary voltage at bus 1043 . The LTC reached its minimum tap at $t=549.6 \mathrm{~s}$, but due to the voltage stability restoration process taking place at nearby buses the moving average increases above the reference value and the setpoint reduction is inhibited. The same holds for the other LTC that met lower tap limits at bus 1044. In Table IX the steps of the LTC control range restoration algorithm are shown for the above two buses.

\section{Generator Voltage Regulation Restoration}

Another interesting feature of Case 2 is that all generators with activated OELs eventually return to normal AVR control. This implies that the protection scheme curtailed more load than the exact amount needed to restore a sta- 
ble equilibrium with limited generators. This is reasonable to expect, as the contingency is only marginally unstable and thus the minimum load shedding is too small to estimate accurately with some security margin.

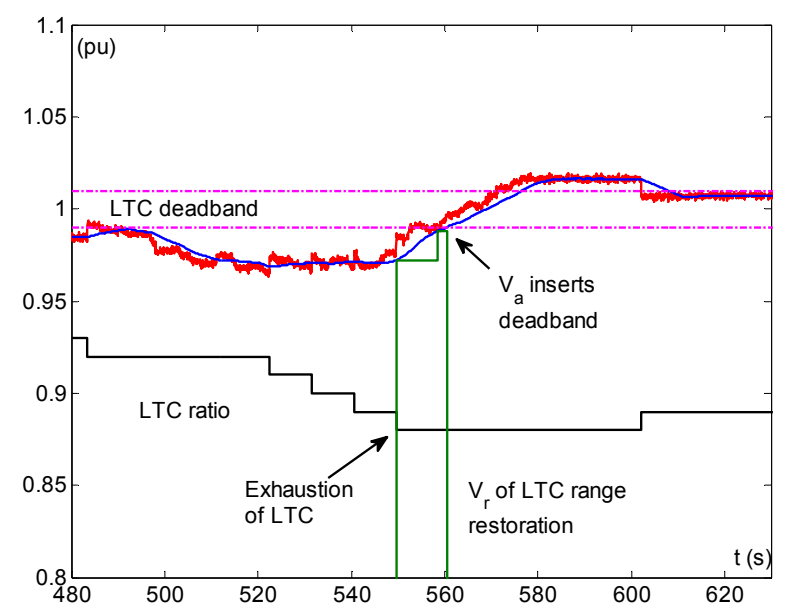

Figure 17: Voltage measurement, moving average, reference voltage and ratio at $\mathrm{HV}$ side of bus 1043, Case 2

Table IX: Sequence of events of LTC range restoration, Case 2

\begin{tabular}{|c|c|c|c|c|c|}
\hline \multirow{4}{*}{ Bus } & $\boldsymbol{t}$ (s) & $\boldsymbol{V}_{\boldsymbol{a}}$ & $\boldsymbol{V}_{\boldsymbol{r}}$ & Action & $\begin{array}{c}\boldsymbol{V}_{\text {set }} \\
\text { reduc- } \\
\text { tion }\end{array}$ \\
\hline \multirow{4}{*}{1043} & 549.6 & 0.972 & 0.972 & $V_{r}=V_{a}$ & \\
\cline { 2 - 7 } & 558.6 & 0.988 & & $V_{a}>V_{r}$ & inhibit \\
\cline { 2 - 6 } & 558.6 & 0.988 & 0.988 & $V_{r}=V_{a}$ & \\
\cline { 2 - 6 } & 560.5 & 0.99 & & deadband & STOP \\
\hline \multirow{5}{*}{1044} & 551.1 & 0.973 & 0.973 & $V_{r}=V_{a}$ & \\
\cline { 2 - 6 } & 561.1 & 0.989 & & $V_{a}>V_{r}$ & inhibit \\
\cline { 2 - 6 } & 561.1 & 0.989 & 0.989 & $V_{r}=V_{a}$ & \\
\cline { 2 - 6 } & 562.0 & 0.99 & & deadband & STOP \\
\hline
\end{tabular}

As seen in Table VII the OEL of generator g6 is activated at $t=556.9 \mathrm{~s}$, only a few seconds before the first restore signal at $t=560 \mathrm{~s}$. This more or less accidental event increased the total amount of indirect load shedding necessary to achieve stability.

In Fig. 18 the control (error) signals of OEL and AVR are shown for generator $\mathrm{g} 6$. The control mode depends on the comparison of these two signals. The AVR error is $V_{c}-V_{t}$, corresponding to the difference between the reference and actual value of terminal voltage, while the OEL error $I_{\text {flim }}{ }^{-}$ $I_{f}$ corresponds to the difference between the limit and measured value of the generator field current. When the former error is smaller, the generator operates under AVR control; otherwise it switches under OEL control with a specified time delay different for each generator.

In Fig. 19 the active load demand and consumption are shown. It is clear that the total indirect load shedding is more than the minimum, as at $t=575$ s the load consumption becomes larger than the load demand due to the succession of generators switching back under AVR control.

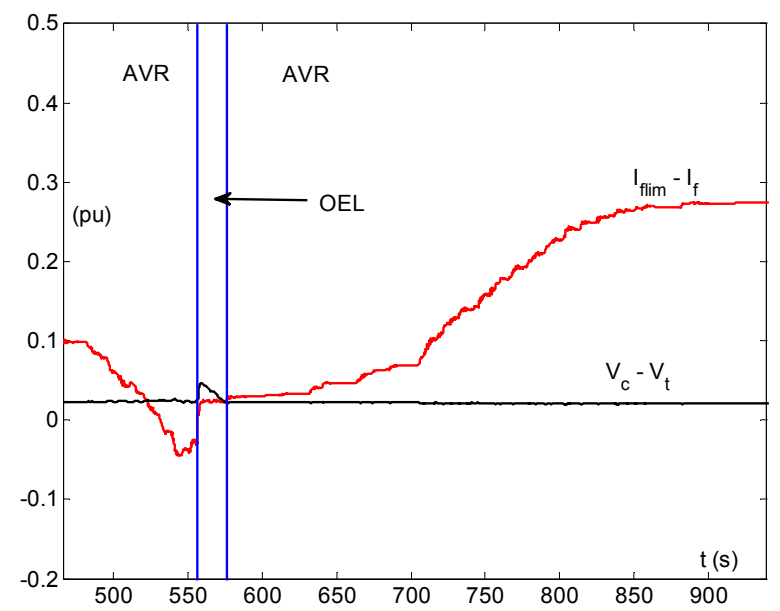

Figure 18: OEL and AVR signals of generator g6, Case 2

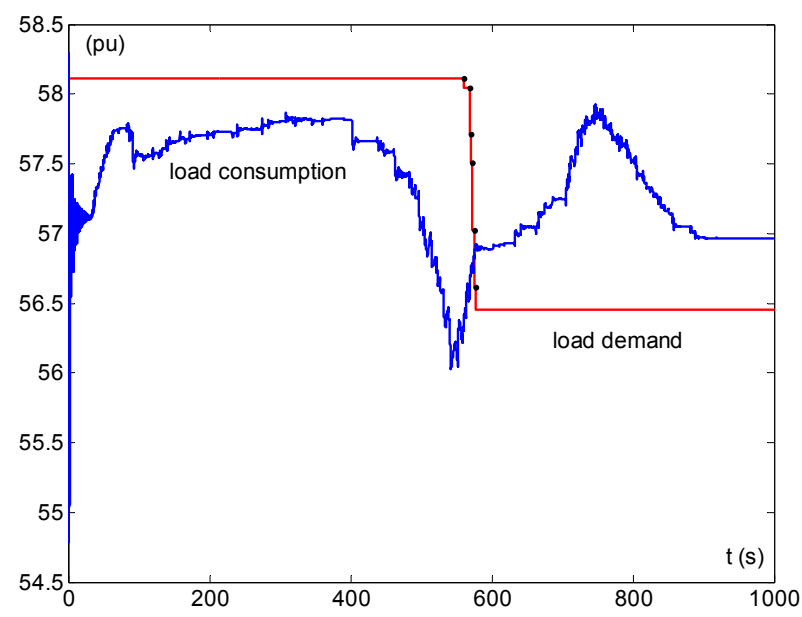

Figure 19: Total active load consumption and demand of the Central Area, Case 2

\section{Conclusions}

This paper presented an integrated and autonomous protection scheme that can be implemented on a simple LTC controller using only locally available signals. The protection is based on the LIVES detection method introduced in previous publications.

The integrated protection system consists of three different application modules that were explained and tested in the paper. In the first module the LIVES method is used as in previous publications to identify the onset of voltage instability and issue an alarm. This module was named LIVES-alarm. The second module introduced in this paper in order to restore voltage stability consists of a series 
of reverse tap actions that continues until a stability condition is monitored, after which the setpoint of the controlled secondary LTC voltage is reduced to its measured value. This module was called LIVES-restore. The identification of stability conditions was achieved with a variant of the LIVES method monitoring the primary (transmission-side) voltage of the LTC during reverse tap actions. The third module consists of the restoration of controllability when LTCs reach their lower tap limits. This module monitors the secondary voltage when the lower tap is reached and performs a secondary voltage setpoint reduction, when this voltage is below the deadband and keeps decreasing. This third module is necessary, because it allows LIVES-alarm function to operate during long incidents where LTC tap range is exhausted.

Detailed simulation showed that the proposed scheme was able to protect the system in two unstable test cases, manifesting severe and marginal instability respectively.

The proposed integrated autonomous protection system was compared to direct load shedding schemes and in particular to the minimum load shedding achieved by a global monitoring method. The results of this comparison were very promising. Although the total amount of load shedding was larger when using the LIVES algorithm, the difference is not considered significant, when taking into account that a security margin is always necessary. Furthermore, the proposed protection scheme has the advantage of being easily installed in existing apparatus, without the need for communication, or wiring and arming of selected feeder breakers to achieve the required firm load shedding.

Concerning the LTC control range restoration module, it was not activated during the simulation runs, but its operation was tested in Case 2, where it was seen that its operation was correctly inhibited due to the rising secondary voltages.

Further research is needed to assess the performance and improve the tuning of the LTC control range restoration module. Finally more simulations need to be carried out with self restoring loads in order to clarify the behavior of the proposed integrated autonomous protection scheme in this case.

\section{References}

[1] C. Vournas, N. Sakellaridis, "Tracking Maximum Loadability Conditions in Power Systems", Bulk Power System Dynamics and Control-VII, Charleston, SC, USA, Aug. 2007.

[2] C. D. Vournas, T. Van Cutsem, "Local Identification of Voltage Emergency Situations", IEEE Trans. on Power Systems, Vol. 23, no. 3 , pp. 1239 - 1248, Aug. 2008.

[3] C. Vournas, N. Sakellaridis, G. Christoforidis, J. Kabouris, T. Van Cutsem, "Investigation of a Local Indicator of Voltage Emergency in the Hellenic Interconnected System", 16th PSCC, Glasgow, July 2008.

[4] C. D. Vournas, N. G. Sakellaridis, "Problems and Solutions for Local Identification of Voltage Instability and Emergency Control”, IEEE/PES General Meeting, Pittsburgh, PA, July 2008.

[5] K. Vu, M. M. Begovic, D. Novosel, M. M. Saha, "Use of local measurements to estimate voltage stability margins", IEEE Trans. on Power Systems, Vol. 14, 1999, pp. 1029-1035

[6] A. Holen, L. Warland, "Estimation of distance to voltage collapse: testing an algorithm based on local measurements", Proc. 14th Power System Computation Conference, Seville, 2002, paper 38-3.

[7] S. Corsi, G. N. Taranto, "A Real-Time Voltage Instability Identification Algorithm Based on Local Phasor Measurements," IEEE Trans. on Power Syst., Vol. 23,No. 3, pp. 1271-1279, Aug. 2008

[8] CIGRE TF 38.02.08 (M. Stubbe, convenor), "Long-term dynamics - Phase II", Final report, January 1995.

[9] M. Glavic, T. Van Cutsem, "Wide-Area Detection of Voltage Instability From Synchronized Phasor Measurements. Part I: Principle", IEEE Trans. on Power Systems, Vol. 24, no. 3, pp. 1408 1416, Aug. 2009.

[10] M. Glavic, T. Van Cutsem, "Wide-Area Detection of Voltage Instability From Synchronized Phasor Measurements. Part II: Simulation Results", IEEE Trans. on Power Systems, Vol. 24, no. 3, pp. 1417 - 1425, Aug. 2009.

[11] C. D. Vournas and N. G. Sakellaridis, "Region of Attraction in a Power System with Discrete LTCs", IEEE Trans. on Circuits \& Systems I, vol. 53, pp. 1610--1618, July 2006.

[12] J. Medanic, M. Ilic-Spong, and J. Christensen, "Discrete Models of slow voltage dynamics for under load tap-changing transformer coordination", IEEE Trans. on Power Systems, vol. 2, pp. 873-882, Nov. 1987.

[13] F. Capitanescu, T. Van Cutsem, "Unified sensitivity analysis of unstable or low voltages caused by load increases or contingencies," IEEE Trans. Power Syst., Vol. 20, No. 1, pp. 321-329, Feb. 2005. 